Gut and Liver, Vol. 9, No. 4, July 2015, pp. 486-493

\title{
The Effects of Broccoli Sprout Extract Containing Sulforaphane on Lipid Peroxidation and Helicobacter pylori Infection in the Gastric Mucosa
}

\author{
Young Woon Chang*, Jae Young Jang*, Yong Ho Kim ${ }^{\dagger}$, Jung-Wook Kim*, and Jae-Jun Shim* \\ Departments of *Internal Medicine and ${ }^{\dagger}$ Surgery, Kyung Hee University College of Medicine, Seoul, Korea
}

Background/Aims: The aims of this study were to investigate whether a broccoli sprout extract containing sulforaphane (BSES) inhibited the Helicobacter pylori infection density and exerted an antioxidative effect on gastric mucosal damage. Methods: The enrolled subjects were randomized in a double-blinded manner into three groups. Finally, 33 H. pylori (+) BSES treatment subjects (group A), $28 \mathrm{H}$. pylori (+) placebo subjects (group B), and $28 \mathrm{H}$. pylori (-) BSES treatment subjects (group $\mathrm{C}$ ) were studied. $H$. pylori infection density was indirectly quantified by a ${ }^{13} \mathrm{C}$-urea breath test (UBT), and the ammonia concentration in gastric juice aspirates was measured through gastroscopic examination. Malondialdehyde (MDA), an oxidative damage biomarker, and reduced glutathione (GSH), an antioxidant biomarker, were measured in the gastric mucosa by an enzyme-linked immunosorbent assay. Results: BSES treatment did not significantly affect the UBT values or ammonia concentration in group $A(p=0.634$ and $p=0.505$, respectively). BSES treatment did significantly reduce mucosal MDA concentrations in group $A(p<0.05)$ and group $C(p<0.001)$, whereas the gastric mucosal GSH concentrations did not differ before and after treatment in any of the groups. Conclusions: BSES did not inhibit the $H$. pylori infection density. However, BSES prevented lipid peroxidation in the gastric mucosa and may play a cytoprotective role in $\mathrm{H}$. pylori-induced gastritis. (Gut Liver 2015;9:486-493)

Key Words: Helicobacter pylori; Sulforaphane; Malondialdehyde; Glutathione

\section{INTRODUCTION}

Helicobacter pylori infects approximately 50\% of the adult population worldwide and is associated with gastritis, peptic ul- cer disease, and gastric cancer. ${ }^{1}$ In Korea, the $H$. pylori infection rate in adults 16 to 79 years is approximately $60 \%,{ }^{2}$ and gastric cancer is the second most frequently diagnosed malignancy. ${ }^{3}$ Successful eradication of $H$. pylori could be beneficial in alleviating $H$. pylori-related gastroduodenal diseases and reduce the risk of gastric cancer in countries such as Korea, which have a high prevalence of $H$. pylori infection and high incidence of gastric cancer.

Proton pump inhibitor (PPI)-based triple therapy is the most effective treatment for $H$. pylori eradication, but has become less successful, with eradication rates as low as 50\% to $70 \%$, due to low compliance and high rates of antibiotic resistance. ${ }^{4,5}$ The standard eradication therapy for $H$. pylori infection involves a 1-week triple therapy, combining a PPI with two antibiotics (i.e., clarithromycin plus amoxicillin or metronidazole). ${ }^{6,7}$ However, primary and acquired metronidazole or clarithromycin resistance has been discovered worldwide, resulting in treatment failure when using regimens including these antibiotics. In Korea, resistance to metronidazole and clarithromycin has been reported in $66.2 \%$ and $38.5 \%$ of patients, respectively. ${ }^{8}$ In addition, high rates of antibiotic-associated side effects may result in poor patient compliance. ${ }^{9}$ Therefore, it is necessary to develop new treatment strategies that increase the eradication rate and reduce adverse effects.

Gastric mucosal damage in $H$. pylori-associated gastritis could be caused directly by $H$. pylori or as a consequence of the inflammatory reaction. Oxygen free radicals are released by polymorphs and other inflammatory cells in $H$. pylori infection, ${ }^{10}$ initiating lipid peroxidation by oxygen free radicals. Both oxygen free radical formation and lipid peroxidation are strongly linked to tissue damage and may play a role in the multistep pathogenesis of chronic lesions and possibly of cancer. ${ }^{11}$

Sulforaphane is a molecule within the isothiocyanate group of organosulfur compounds. It is obtained from cruciferous

Correspondence to: Jae Young Jang

Department of Gastroenterology, Kyung Hee University College of Medicine, 26 Kyungheedae-ro, Dongdaemun-gu, Seoul 130-701, Korea

Tel: +82-2-958-8200, Fax: +82-2-958-1848, E-mail: jyjang@khu.ac.kr

Received on February 3, 2014. Revised on May 14, 2014. Accepted on May 26, 2014. Published online October 7, 2014

pISSN 1976-2283 eISSN 2005-1212 http://dx.doi.org/10.5009/gnl14040

@ This is an Open Access article distributed under the terms of the Creative Commons Attribution Non-Commercial License (http://creativecommons.org/licenses/by-nc/4.0) which permits unrestricted non-commercial use, distribution, and reproduction in any medium, provided the original work is properly cited. 
vegetables such as broccoli, Brussels sprouts, or cabbages. Sulforaphane has strong bactericidal activity against $H$. pylori in vitro. ${ }^{12}$ Moreover, sulforaphane is highly active against a large number of clinical isolates of $H$. pylori, many of which are resistant to conventional antibiotics. ${ }^{13}$ Recently, broccoli sprout extract containing sulforaphane (BSES) was reported to reduce colonization and attenuate gastritis in $H$. pylori-infected mice and humans. ${ }^{14}$ Sulforaphane is also a potent inducer of phase 2 detoxification enzymes, such as glutathione S-transferase, and exhibits antioxidative, anti-inflammatory, and anticancer effects. ${ }^{15-17}$ Glutathione S-transferases catalyze the conjugation of glutathione (GSH) to xenobiotic substrates for detoxification. Therefore, intracellular GSH is one of the preventive factors against oxidative damage. ${ }^{18}$

This prospective study investigated whether BSES inhibited $H$. pylori infection and stimulated an antioxidative effect on inflamed gastric mucosa in patients with $H$. pylori-infected functional dyspepsia.

\section{MATERIALS AND METHODS}

\section{Subjects and study design}

One hundred volunteer subjects with functional dyspepsia were randomized double blindly from March 2009 to October 2011. Medical history interviews were conducted in all participants, followed by a brief physical examination. Exclusion criteria were previous gastric surgery, peptic ulcer disease, gastric malignancy, use of nonsteroidal anti-inflammatory drugs or anticoagulant drugs, and systemic diseases such as diabetes, hypertension, and heart disease. We included the subjects with nonatrophic erythematous gastritis (mild to moderate) in accordance with the updated Sydney system to avoid the bias of divergent gastritis patterns. ${ }^{19}$

We performed upper gastrointestinal endoscopy with measurements of urea breath test (UBT), malondialdehyde (MDA), and GSH concentration twice before and after intervention in all subjects. Follow-up examinations were performed within 1 week after taking all the BSES capsules. After an overnight fast, upper gastrointestinal endoscopy and a UBT were performed. Sixty-seven of the 100 subjects were $H$. pylori positive. Subjects with $H$. pylori infection were randomized double blindly to receive either one BSES capsule containing a 250-mg standardized broccoli sprout yielding 1,000 $\mu$ g sulforaphane (Oregon Health, Phoenix, AZ, USA) or placebo twice daily for 4 weeks. The placebo capsules were identical to the BSES capsules in appearance. Thirty-four of the $67 \mathrm{H}$. pylori (+) subjects received BSES and 33 subjects received the placebo. The 33 subjects who were $H$. pylori (-) received BSES. One of the $34 \mathrm{H}$. pylori (+) BSES subjects, five of the $33 \mathrm{H}$. pylori (+) placebo subjects, and five of the $33 \mathrm{H}$. pylori (-) BSES subjects were lost during follow-up. Therefore, the study comprised 89 subjects: 33 in the H. pylori (+) BSES group (group A), $28 H$. pylori (+) in the placebo group (group B), and $28 \mathrm{H}$. pylori (-) in the BSES group (group C). Informed consent was obtained from all individuals before entering the study. This study was approved by the Institutional Review Board of Kyung Hee University Hospital (KMC IRB 0401-0410).

\section{2. ${ }^{13} \mathrm{C}$-UBT}

In all instances, the ${ }^{13} \mathrm{C}$-UBT was performed on the first day after an overnight fast or at least after an 8-hour fast. A baseline breath sample was collected into a collection tube. An aliquot of $75 \mathrm{mg}{ }^{13} \mathrm{C}$-urea dissolved in $75 \mathrm{~mL}$ of citric acid solution was given orally (Helikit; Isodiagnostika, Edmonton, Canada). Another breath sample was collected after 30 minutes. Breath samples were subsequently analyzed by mass spectrometry to determine the ${ }^{13} \mathrm{C} /{ }^{12} \mathrm{C}$ ratio (HeliView; MediChems, Seoul, Korea). The ${ }^{13} \mathrm{C} /{ }^{12} \mathrm{C}$ ratio of each breath sample was expressed as a millipercentage (\%o). Changes in the ${ }^{13} \mathrm{C}$ value compared to baseline were expressed as $\Delta^{13} \mathrm{C}$. A positive result was defined as an increase $>4 \%$.

\section{Gastroscopic examination}

Eighty-nine subjects underwent gastroscopy before and after BSES or placebo treatment. Upon entering the stomach, 10 to $20 \mathrm{~mL}$ of gastric juice were aspirated from the gastric fundus and collected in a trap. During the gastroscopic examination, one biopsy specimen was taken from the antrum, and four pieces were taken from the lower gastric corpus. The antral and one corpus biopsy specimen were submitted for a rapid urease test (CLO test; Green Cross Co., Seoul, Korea). The three remaining corpus mucosal tissues were used for measurement of MDA and GSH concentrations.

\section{Ammonia concentration in gastric Juice}

The ammonia concentration in gastric juice was used as an additional indicator of $H$. pylori colony density in the gastric mucosa. We used a timed endpoint method to determine ammonia concentration based on a reaction between $\mathrm{NH}_{3}, \mathrm{NADPH}$ and 2-oxoglutarate in the presence of the enzyme glutamate dehydrogenase. The stoichiometry of the reaction can be determined by NADPH disappearance. Aspirated gastric juice was frozen at $-70^{\circ} \mathrm{C}$ until analysis. After thawing, the gastric juice specimen was centrifuged at 3,000xg for 15 minutes to separate gastric mucus and debris. The centrifuged samples were diluted 1:10 in Tris buffer (pH 7.2) and the concentration of ammonia in the samples determined from spectrophotometric readings at $340 \mathrm{~nm}$ (Synchron LX20; Beckman Coulter, Fullerton, CA, USA). ${ }^{20}$

\section{Indirect quantification of $\boldsymbol{H}$. pylori infection density}

H. pylori infection was diagnosed upon a positive result in the rapid urease test and ${ }^{13} \mathrm{C}$-UBT. The successful eradication of $H$. pylori was defined as a decrease to $<4 \%$ in the ${ }^{13} \mathrm{C}$-UBT. Also, $H$. 
pylori infection density was quantified indirectly by a ${ }^{13} \mathrm{C}-\mathrm{UBT}$ and measurement of the ammonia concentration in gastric juice aspirate. $^{21}$

\section{GSH concentration as an antioxidant biomarker in the gastric mucosa}

The measurement of GSH was based on a chemical reaction that proceeds in two steps. The first step leads to the formation of thioethers between 4-chloro-1-methyl-7-trifluromethylquinolinium-methylsulfate and mercaptans. The second step is a $\beta$-elimination reaction, which takes place under alkaline conditions. Thioether reacts with GSH to form a chromophoric thione. Biopsy specimens from the gastric body mucosa for $\mathrm{GSH}$ determination were immediately frozen and stored at $-70^{\circ} \mathrm{C}$ until analysis. Each sample was weighed and placed into $20 \mathrm{~mL}$ of 5-metaphosphoric acid for homogenization of the tissue. The homogenate was centrifuged at 3,000×g for 10 minutes and GSH concentration measured by colorimetric assay at $400 \mathrm{~nm}$ (Bioxytech GSH-400; Oxis, Portland, OR, USA). ${ }^{22}$

\section{MDA concentration as an oxidative damage biomarker in gastric mucosa}

Measurement of MDA is widely used as an indicator of lipid peroxidation due to cellular injury. The principle is based on the reaction of a chromogenic reagent, $N$-methyl-2-phenylindole with MDA at $45^{\circ} \mathrm{C}$ for 1 hour. $N$-Methyl-2-phenylindole reacts

Table 1. Basic Clinical Characteristics of the Subjects

\begin{tabular}{lcccc}
\hline \multicolumn{1}{c}{ Characteristic } & $\begin{array}{c}\text { Group A } \\
(\mathrm{n}=33)\end{array}$ & $\begin{array}{c}\text { Group B } \\
(\mathrm{n}=28)\end{array}$ & $\begin{array}{c}\text { Group C } \\
(\mathrm{n}=28)\end{array}$ & p-value \\
\hline Male/female & $16 / 17$ & $14 / 14$ & $13 / 15$ & 0.965 \\
Age & $39.2 \pm 11.4$ & $42.3 \pm 11.2$ & $36.1 \pm 11.7$ & 0.138 \\
Alcohol & $19(57.6)$ & $18(64.3)$ & $16(57.1)$ & 0.826 \\
Smoking habits & $10(30.3)$ & $8(28.6)$ & $5(17.9)$ & 0.501 \\
\hline
\end{tabular}

Data are presented as mean \pm SD or number $(\%)$.

Group A, Helicobacter pylori (+) broccoli sprout extract containing sulforaphane (BSES) treatment group; Group B, H. pylori (+) placebo treatment group; Group $\mathrm{C}, H$. pylori (-) BSES treatment group. with MDA to form an intensely colored carbocyanine dye. Biopsy specimens from the gastric body mucosa for MDA determination were immediately frozen and stored at $-70^{\circ} \mathrm{C}$ until analysis. Each sample was weighed and placed into $20 \mathrm{~mL}$ of $0.5 \mathrm{M}$ butylated hydroxytoluene for homogenization. The homogenate was centrifuged at 3,000xg for 10 minutes, and the MDA concentration measured by colorimetric assay at $586 \mathrm{~nm}$ (Bioxytech LPO-586; Oxis). ${ }^{23}$

\section{Statistical analysis}

All data were analyzed using SPSS version 18.0 (SPSS Inc., Chicago, IL, USA). All data were expressed as means \pm standard deviation (SD). Between-group differences were assessed by means of one-way analysis of variance for continuous variables and Fisher exact test for categorical variables. Comparison of the clinicopathological features was assessed using a two tailed chi-square test and independent sample t-test. In the analysis of MDA, GSH, UBT, and ammonia concentrations before and after BSES or placebo treatment, a paired t-test was used.

\section{RESULTS}

\section{Clinical characteristics}

There were no significant differences in basic clinical features between the $H$. pylori (+) BSES group and the $H$. pylori (+) placebo group. However, the $H$. pylori (-) BSES group was younger with fewer smokers (Table 1).

\section{Inhibitory effect on $H$. pylori}

In the $H$. pylori $(+)$ BSES group ( $\mathrm{n}=33)$, there were no significant differences in $\triangle \mathrm{UBT}$ values between before and after treatment $(\mathrm{p}=0.634)$ (Table 2). In only one subject, the $\Delta \mathrm{UBT}$ value was reduced to $<50 \%$ of the basal level. There were no significant differences in ammonia concentrations before and after treatment ( $p=0.505)$ (Table 2). Additionally, the ammonia concentrations in gastric juice were not reduced to $<50 \%$ of the basal level in the $H$. pylori (+) BSES or $H$. pylori (+) placebo group.

Table 2. Inhibitory Effect of Broccoli Sprout Extract Containing Sulforaphane on Helicobacter pylori Infection

\begin{tabular}{|c|c|c|c|c|c|c|c|}
\hline & \multicolumn{3}{|c|}{ Group A (n=33) } & \multicolumn{3}{|c|}{ Group B (n=28) } & \multirow{2}{*}{ p-value* } \\
\hline & Before & After & $\mathrm{p}$-value & Before & After & p-value & \\
\hline$\Delta \mathrm{UBT}, \%$ & $60.5 \pm 25.6$ & $58.3 \pm 23.8$ & 0.531 & $59.7 \pm 28.2$ & $62.7 \pm 25.4$ & 0.329 & 0.601 \\
\hline Ammonia, mmol/L & $9.3 \pm 4.4$ & $9.6 \pm 4.5$ & 0.505 & $10.1 \pm 5.7$ & $10.7 \pm 5.5$ & 0.224 & 0.270 \\
\hline $50 \%$ decrease of $\Delta \mathrm{UBT}$ value & 32 & $1^{\dagger}$ & - & 28 & 0 & - & - \\
\hline $50 \%$ decrease of ammonia level & 33 & 0 & - & 28 & 0 & - & - \\
\hline
\end{tabular}

Data are presented as mean \pm SD or number.

UBT, urea breath test.

${ }^{*}$ p-values were calculated by an independent t-test between groups A and B; ${ }^{\dagger}$ In only one subject in group A was the $\Delta$ UBT value below $50 \%$ of the basal level. 


\section{Inhibition of lipid peroxidation and antioxidative effect of BSES}

There were no statistically significant differences in basal mucosal MDA $(\mathrm{p}=0.083)$ and GSH $(\mathrm{p}=0.526)$ concentrations between the H. pylori $(+)(\mathrm{n}=61)$ and $H$. pylori $(-)(\mathrm{n}=28)$ groups (Table 3). After BSES treatment, mucosal MDA concentrations were significantly decreased in $H$. pylori (+) BSES subjects compared to those before treatment $(n=33, p=0.006)$ (Table 4, Fig. 1). Furthermore, MDA concentrations were significantly decreased

Table 3. Basal Malondialdehyde and Glutathione Concentrations in Helicobacter pylori-Positive and -Negative Subjects

\begin{tabular}{lccc}
\hline & $\begin{array}{c}\text { All H. pylori (+) } \\
\text { groups } \\
\left(\begin{array}{c}\text { Group A+B) } \\
(\mathrm{n}=61)\end{array}\right.\end{array}$ & $\begin{array}{c}\text { H. pylori (-) } \\
\text { group } \\
\left(\begin{array}{c}\text { Group C) } \\
(\mathrm{n}=28)\end{array}\right.\end{array}$ & p-value \\
\hline Basal MDA, nmol/g tissue & $151.4 \pm 109.2$ & $112.7 \pm 60.9$ & 0.083 \\
Basal GSH, $\mu \mathrm{mol} / \mathrm{g}$ tissue & $102.0 \pm 60.8$ & $111.4 \pm 66.4$ & 0.526 \\
\hline
\end{tabular}

Data are presented as mean \pm SD.

MDA, malondialdehyde; GSH, glutathione.
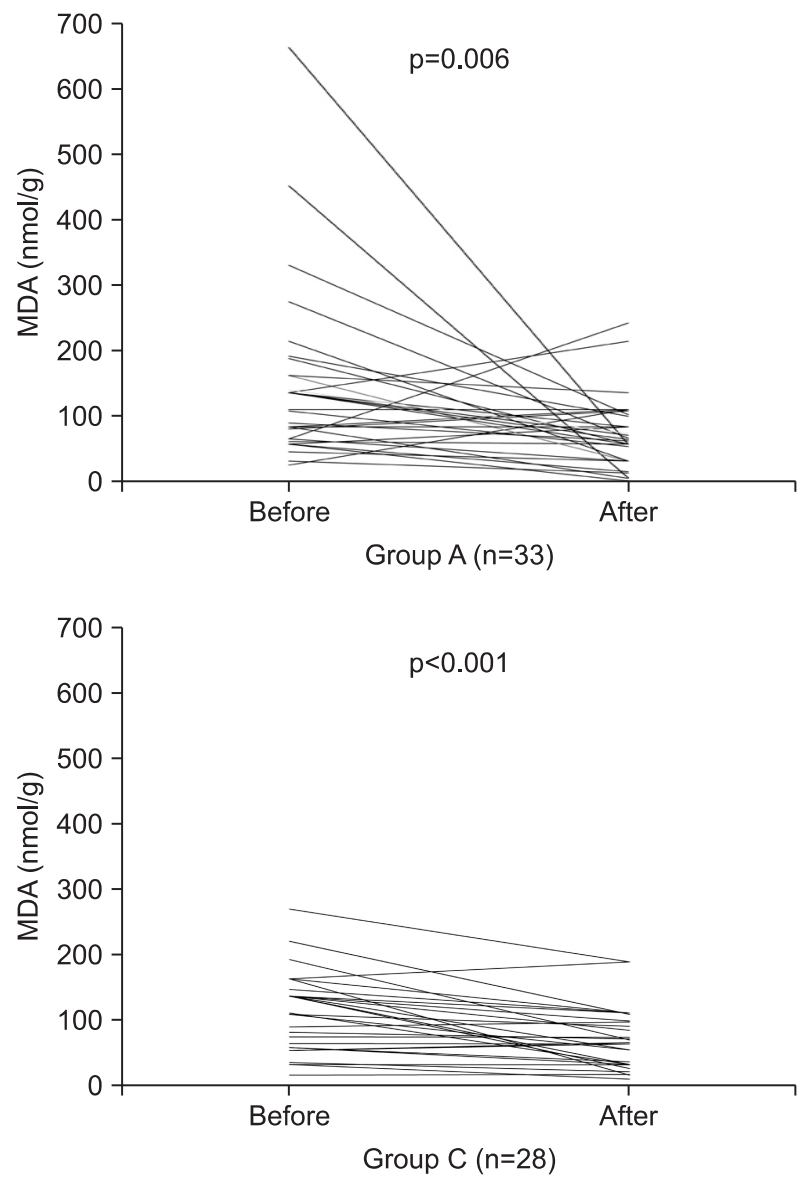

in all BSES treatment subjects $(n=61)$, irrespective of $H$. pylori infection $(\mathrm{p}<0.001)$ (Table 4).

Table 4. Antioxidative Effect of Broccoli Sprout Extract Containing Sulforaphane and Placebo in All Subjects

\begin{tabular}{lcccc}
\hline & Before & After & $\Delta \mathrm{D}$ & p-value \\
\hline MDA, nmol/g & & & & \\
Group A (n=33) & $147.8 \pm 127.8$ & $74.7 \pm 53.1$ & $-73.0 \pm 143.1$ & 0.006 \\
Group B (n=28) & $155.7 \pm 84.3$ & $144.8 \pm 57.3$ & $-10.9 \pm 53.3$ & 0.289 \\
Group C ( $\mathrm{n}=28)$ & $112.7 \pm 60.9$ & $71.7 \pm 46.1$ & $-41.0 \pm 46.5$ & $<0.001$ \\
GSH, $\mu$ mol/g & & & & \\
Group A (n=33) & $99.8 \pm 70.5$ & $109.9 \pm 82.5$ & $10.1 \pm 91.8$ & 0.532 \\
Group B (n=28) & $104.5 \pm 48.0$ & $110.3 \pm 47.4$ & $5.8 \pm 35.1$ & 0.391 \\
Group C (n=28) & $111.4 \pm 66.4$ & $118.5 \pm 64.3$ & $7.1 \pm 27.0$ & 0.178 \\
\hline
\end{tabular}

Data are presented as mean \pm SD. $\Delta \mathrm{D}$ was the difference before and after treatment. $\mathrm{p}$-values were calculated by paired t-tests, before and after treatment, in each group. When comparing the $\Delta \mathrm{D}$ between groups $\mathrm{A}$ and $\mathrm{B}$, the $\mathrm{p}$-values were 0.026 for MDA and 0.804 for $\mathrm{GSH}$. When comparing the $\triangle \mathrm{D}$ between group $\mathrm{A}+\mathrm{C}$ and group $\mathrm{B}$, the p-values were 0.034 for MDA and 0.834 for GSH. MDA, malondialdehyde; GSH, glutathione.
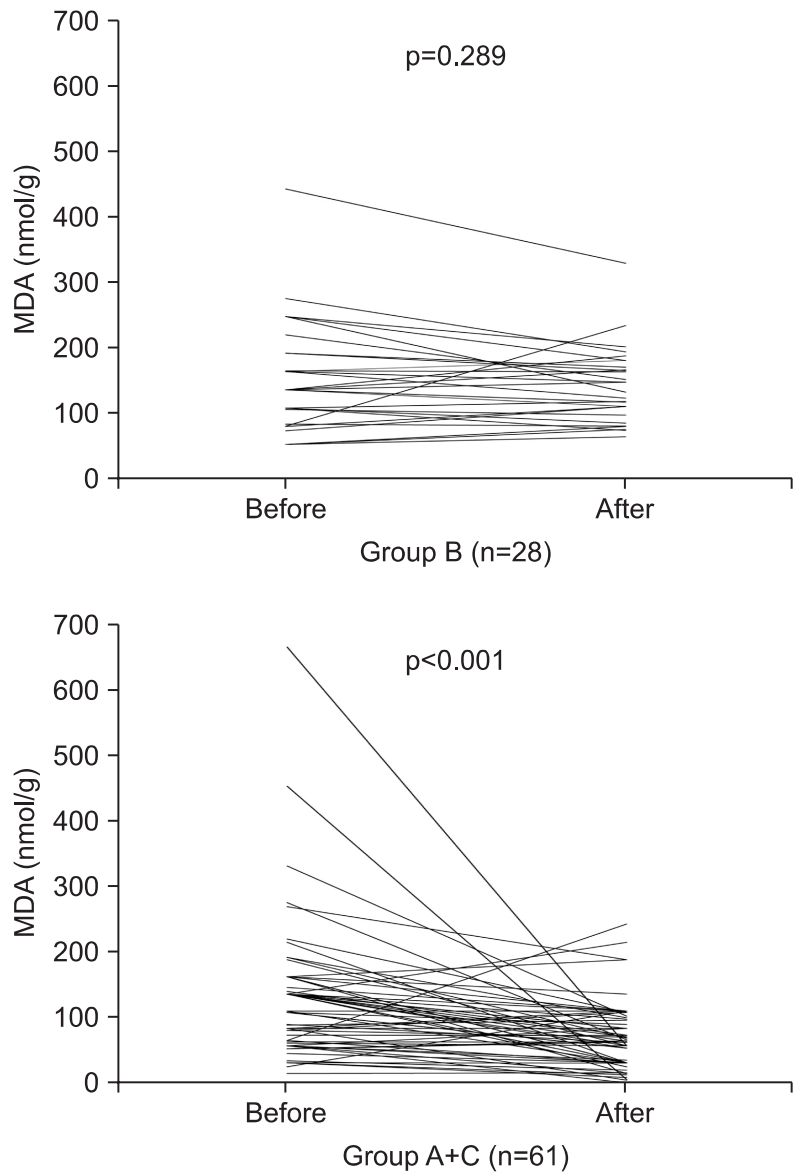

Fig. 1. Comparison of malondialdehyde (MDA) concentrations in the gastric mucosal tissue before and after treatment in each group. After broccoli sprout extract containing sulforaphane (BSES) treatment, the mucosal MDA concentrations were significantly decreased in group A compared with those before treatment $(\mathrm{p}=0.006)$. Furthermore, the MDA concentrations were significantly decreased in all BSES treatment subjects (group $\mathrm{A}+\mathrm{C})$, regardless of Helicobacter pylori infection status $(\mathrm{p}<0.001)$. 


\section{Antioxidative effect of BSES}

In all $61 \mathrm{H}$. pylori (+) subjects, basal GSH concentrations were lower than those of the $28 \mathrm{H}$. pylori (-) subjects. However, the differences were not significant $(\mathrm{p}=0.526)$ (Table 3). GSH concentrations were increased slightly after BSES treatment, irrespective of $H$. pylori infection status ( $\mathrm{p}=0.332)$ (Table 4, Fig. 2).

\section{DISCUSSION}

One of the alternative treatments for $H$. pylori infection is probiotics, such as lactic acid bacteria and bifidobacteria. Administration of probiotics improves $H$. pylori eradication rates and reduces the side effects of first-line PPI-based triple therapy. ${ }^{24,25}$ However, the inhibitory effect of probiotics on $H$. pylori infection of human remains controversial. For example, specially designed yogurt containing specific probiotics was ineffective for eradication in asymptomatic $H$. pylori-infected women. ${ }^{26}$ Furthermore, the addition of yogurt containing probiotics to second-line treatments containing moxifloxacin neither improved eradication rates nor reduced the adverse effects of treatment. $^{27}$

Broccoli is another candidate supplement for $H$. pylori eradication therapy. Broccoli contains isothiocyanate sulforaphane, which is a potent inducer of phase 2 detoxification enzymes, such as glutathione S-transferase and quinone reductase, that exhibits cellular antioxidative, anti-inflammatory, and anticancer effects. ${ }^{15-17}$ Glutathione S-transferases catalyze the conjugation of GSH to xenobiotic substrates for detoxification. Therefore, intracellular GSH is a preventive factor against oxidative damage. ${ }^{18}$ Sulforaphane extracted from the broccoli sprout is 10 to 100 times more effective in activating phase 2 enzymes than that from mature broccoli. ${ }^{15}$

Sulforaphane is a potent bacteriostatic agent against $H$. pylori in mice and exhibits bactericidal effects in a human epithelial cell line. ${ }^{13}$ One preliminary report showed that oral consumption of broccoli sprouts was temporally associated with $H$. pylori eradication in three of nine patients with chronic active gastritis. ${ }^{28}$ Sulforaphane is highly active against a large number of clinical isolates of $H$. pylori, many of which are resistant to conventional antibiotics, such as clarithromycin and metronidazole. ${ }^{13}$ Recently, it was reported that daily intake of sulfora-
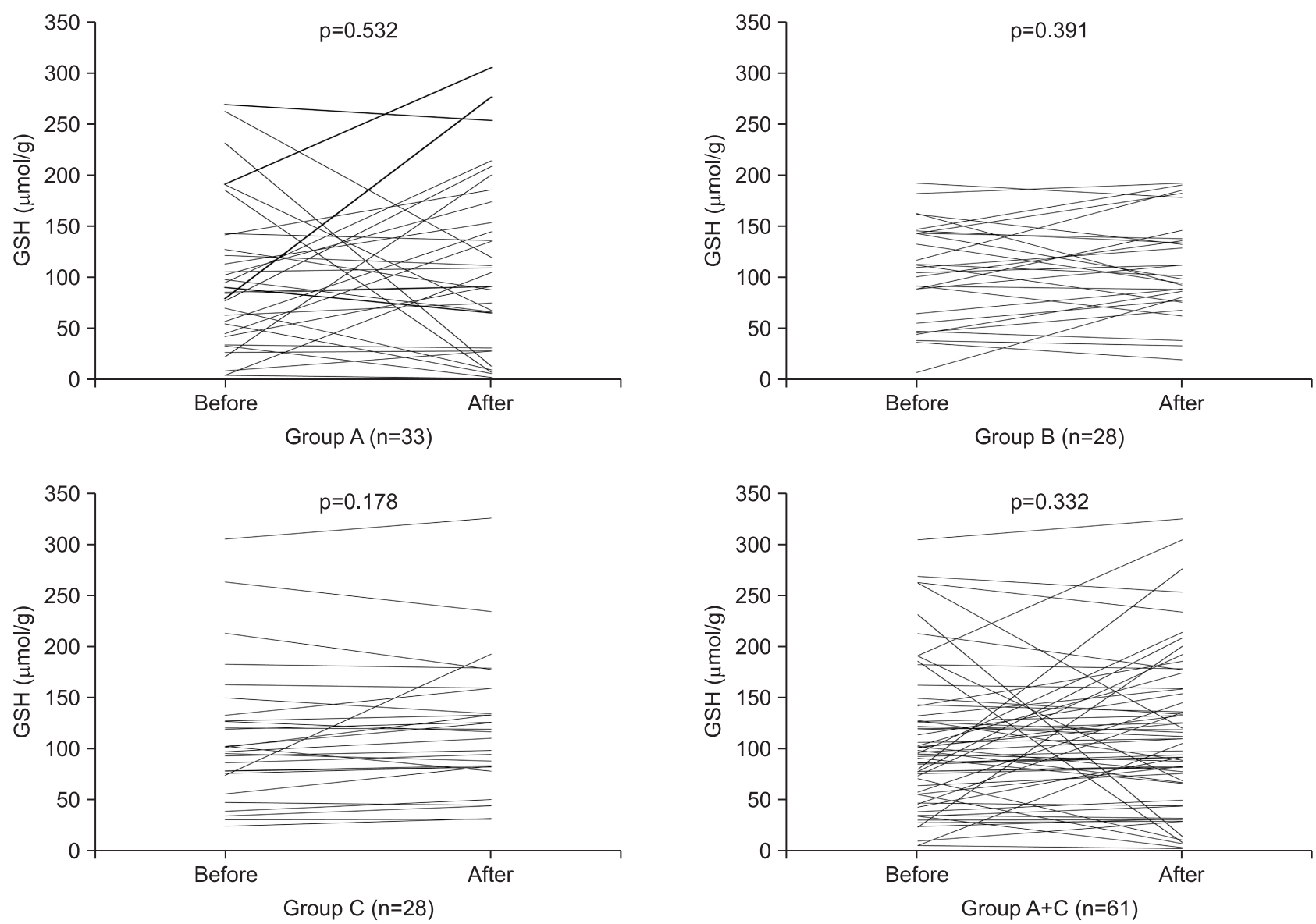

Fig. 2. Comparison of glutathione (GSH) concentrations in the gastric mucosal tissue before and after treatment in each group. There were no significant changes before or after the treatment in any of the three groups. GSH concentrations were increased slightly after broccoli sprout extract containing sulforaphane treatment in the subjects (group $\mathrm{A}+\mathrm{C}$ ), irrespective of their Helicobacter pylori infection status $(\mathrm{p}=0.332$ ). 
phane-rich broccoli sprouts for 2 months reduced $H$. pylori colonization in mice and improved the sequelae of infection in mice and humans. ${ }^{14}$ This treatment appeared to enhance chemoprotection of the gastric mucosa against $H$. pylori-induced oxidative stress via an NrF2-dependent antioxidative and antiinflammatory process. In contrast, an epidemiological study showed that broccoli consumption was not associated with a low prevalence of chronic atrophic gastritis in Japanese males. ${ }^{29}$

In this present study, subjects were instructed to consume BSES twice daily $(2,000 \mu \mathrm{g}$ sulforaphane) for 4 weeks. We measured both the $\triangle \mathrm{UBT}$ value and ammonia concentration in gastric juice as indicators of $H$. pylori colonization inhibition. H. pylori possesses high urease activity and thus produces ammonia from urea in the stomach. Because measurement of ammonia concentration in gastric juices was much simpler and quicker than other methods, it is considered a more valuable method for diagnosing $H$. pylori infection. ${ }^{30} H$. pylori-infected patients had significantly lower urea levels and higher ammonia concentrations as compared with uninfected controls, while gastritis severity increased with gastric juice ammonia concentration. ${ }^{31}$ Thus, measuring the ammonia concentration provides indirect quantitation of the density of $H$. pylori colonies on the gastric mucosa.

We found no significant changes in the $\triangle \mathrm{UBT}$ value and ammonia concentration in gastric juice after sulforaphane treatment and no changes $<50 \%$ of the basal level, except for in one subject. We cannot be certain that 4 weeks is sufficient to achieve any inhibitory effect. Therefore, studies with longer durations of sulforaphane treatment, such as 2 or 6 months, are warranted. Additionally, we plan to examine the effect of sulforaphane supplementation on the efficacy of standard triple first-line or quadruple second-line therapy against $H$. pylori.

We investigated whether sulforaphane had a cytoprotective effect on $H$. pylori-induced gastric mucosal damage. Reactive oxygen radical formation and subsequent lipid peroxidation are the primary mechanisms of human gastric mucosal damage associated with $H$. pylori infection. GSH can scavenge peroxynitrite and hydroxyl radicals, as well as convert hydrogen peroxide to water. Reduced GSH is an active form produced by GSH reductase. ${ }^{32}$ Therefore, reduced GSH reflects the antioxidant effect more accurately than does total GSH. We thus measured reduced GSH concentration in the gastric mucosal tissues as an antioxidant biomarker.

Most human studies have demonstrated that $H$. pylori (+) subjects had a lower mucosal GSH concentration compared with H. pylori (-) subjects. The low GSH concentrations during $H$. pylori infection were presumably because GSH in the gastric mucosa was depleted through removal of oxygen free radicals generated by $H$. pylori. After $H$. pylori eradication, GSH concentrations increased significantly. ${ }^{33-35}$ In this study, H. pylori (+) subjects showed lower GSH concentrations compared to $H$. pylori (-) subjects, but the differences were not significant ( $\mathrm{p}=0.526)$.
It has been suggested that intracellular antioxidants can be consumed by chronic $H$. pylori infection; indeed, BSES treatment for 4 weeks led to slightly increased GSH concentrations in $H$. pylori (+) subjects.

MDA is a product of lipid peroxidation and an indicator of oxidative cell injury. MDA concentrations in the gastric mucosa were significantly higher in subjects with $H$. pylori $(+)$ gastritis than in $H$. pylori (-) subjects and after $H$. pylori eradication. ${ }^{33} \mathrm{In}$ our study, basal MDA concentrations in $H$. pylori (+) subjects were markedly higher than those in $H$. pylori (-) subjects, but the differences did not reach statistical significance $(p=0.083)$. Interestingly, MDA concentrations decreased significantly after BSES treatment in both the H. pylori (+) and H. pylori (-) BSES groups.

The results of this study should be interpreted in the context of several limitations. First, the number of subjects was small, although the antioxidative effect of BSES on gastric mucosal damage was informative. Second, we did not evaluate the long-term (i.e., more than 6 or 12 months) antioxidative, antiinflammatory, and anticancer effects of BSES. Third, we did not compare the relationship between changes in the MDA and GSH concentrations and histological changes in the gastric mucosa. Finally, we did not examine the supplementary effect of BSES on standard triple or quadruple therapy for $H$. pylori eradication.

Although sulforaphane alone had no effect on the eradication or inhibition of $H$. pylori infection, it did reduce the gastric mucosal damage associated with oxidative stress, as measured by MDA, caused by $H$. pylori infection. Our results suggest that sulforaphane may prevent lipid peroxidation in the gastric mucosa and may play a cytoprotective role in $H$. pylori-induced gastritis. However, the relationship between $H$. pylori infection and mucosal GSH concentration was not clear. Further studies using a greater number of subjects and a longer treatment duration are required to confirm these findings.

\section{CONFLICTS OF INTEREST}

No potential conflict of interest relevant to this article was reported.

\section{ACKNOWLEDGEMENTS}

This study was funded by the program of the Kyung Hee University for the young medical researcher in 2007 (KHU20071510).

\section{REFERENCES}

1. McColl KE. Clinical practice. Helicobacter pylori infection. N Engl J Med 2010;362:1597-1604.

2. Kim YS, Baik GH. Epidemiology of Helicobacter pylori infection in 
Korea. Korean J Helicobacter Up Gastrointest Res 2011;11:1-6.

3. Jung KW, Park S, Kong HJ, et al. Cancer statistics in Korea: incidence, mortality, survival, and prevalence in 2009. Cancer Res Treat 2012;44:11-24.

4. Ables AZ, Simon I, Melton ER. Update on Helicobacter pylori treatment. Am Fam Physician 2007;75:351-358.

5. Higuchi K, Maekawa T, Nakagawa K, et al. Efficacy and safety of Helicobacter pylori eradication therapy with omeprazole, amoxicillin and high- and low-dose clarithromycin in Japanese patients: a randomised, double-blind, multicentre study. Clin Drug Investig 2006;26:403-414.

6. Malfertheiner P, Megraud F, O'Morain C, et al. Current concepts in the management of Helicobacter pylori infection: the Maastricht III Consensus Report. Gut 2007;56:772-781.

7. Kim N, Kim JJ, Choe YH, et al. Diagnosis and treatment guidelines for Helicobacter pylori infection in Korea. Korean J Gastroenterol 2009;54:269-278.

8. Kim JG, Kim BJ. Antibiotic resistance in Helicobacter pylori infection. Korean J Helicobacter Up Gastrointest Res 2011;11:13-20.

9. Bell GD, Powell K, Burridge SM, et al. Experience with 'triple' anti-Helicobacter pylori eradication therapy: side effects and the importance of testing the pre-treatment bacterial isolate for metronidazole resistance. Aliment Pharmacol Ther 1992;6:427-435.

10. Dixon MF, Sobala GM. Gastritis and duodenitis: the histopathological spectrum. Eur J Gastroenterol Hepatol 1992;4(Suppl 2):S17-23.

11. Davies GR, Simmonds NJ, Stevens TR, et al. Helicobacter pylori stimulates antral mucosal reactive oxygen metabolite production in vivo. Gut 1994;35:179-185.

12. Basaga HS. Biochemical aspects of free radicals. Biochem Cell Biol 1990;68:989-998.

13. Fahey JW, Haristoy X, Dolan PM, et al. Sulforaphane inhibits extracellular, intracellular, and antibiotic-resistant strains of Helicobacter pylori and prevents benzo[a]pyrene-induced stomach tumors. Proc Natl Acad Sci U S A 2002;99:7610-7615.

14. Yanaka A, Fahey JW, Fukumoto A, et al. Dietary sulforaphanerich broccoli sprouts reduce colonization and attenuate gastritis in Helicobacter pylori-infected mice and humans. Cancer Prev Res (Phila) 2009;2:353-360.

15. Fahey JW, Zhang Y, Talalay P. Broccoli sprouts: an exceptionally rich source of inducers of enzymes that protect against chemical carcinogens. Proc Natl Acad Sci U S A 1997;94:10367-10372.

16. Zhang Y, Talalay P, Cho CG, Posner GH. A major inducer of anticarcinogenic protective enzymes from broccoli: isolation and elucidation of structure. Proc Natl Acad Sci U S A 1992;89:23992403.

17. Barcelo S, Gardiner JM, Gescher A, Chipman JK. CYP2E1-mediated mechanism of anti-genotoxicity of the broccoli constituent sulforaphane. Carcinogenesis 1996;17:277-282.

18. Robert A, Eberle D, Kaplowitz N. Role of glutathione in gastric mucosal cytoprotection. Am J Physiol 1984;247(3 Pt 1):G296-
G304.

19. Dixon MF, Genta RM, Yardley JH, Correa P. Classification and grading of gastritis. The updated Sydney System. International Workshop on the Histopathology of Gastritis, Houston 1994. Am J Surg Pathol 1996;20:1161-1181.

20. Suzuki M, Miura S, Suematsu M, et al. Helicobacter pylori-associated ammonia production enhances neutrophil-dependent gastric mucosal cell injury. Am J Physiol 1992;263(5 Pt 1):G719-G725.

21. Khulusi S, Mendall MA, Patel P, Levy J, Badve S, Northfield TC. Helicobacter pylori infection density and gastric inflammation in duodenal ulcer and non-ulcer subjects. Gut 1995;37:319-324.

22. Anderson ME. Enzymatic and chemical methods for the determination of glutathione. In: Dolphin D, Avramović 0, Poulson R, eds. Glutathione: chemical, biochemical, and medical aspects, part A. New York: John Wiley and Sons, 1989:339-365.

23. Esterbauer H, Schaur RJ, Zollner H. Chemistry and biochemistry of 4-hydroxynonenal, malonaldehyde and related aldehydes. Free Radic Biol Med 1991;11:81-128.

24. Tong JL, Ran ZH, Shen J, Zhang CX, Xiao SD. Meta-analysis: the effect of supplementation with probiotics on eradication rates and adverse events during Helicobacter pylori eradication therapy. Aliment Pharmacol Ther 2007;25:155-168.

25. Bekar 0, Yilmaz Y, Gulten M. Kefir improves the efficacy and tolerability of triple therapy in eradicating Helicobacter pylori. J Med Food 2011;14:344-347.

26. Wendakoon CN, Thomson AB, Ozimek L. Lack of therapeutic effect of a specially designed yogurt for the eradication of Helicobacter pylori infection. Digestion 2002;65:16-20.

27. Yoon H, Kim N, Kim JY, et al. Effects of multistrain probioticcontaining yogurt on second-line triple therapy for Helicobacter pylori infection. J Gastroenterol Hepatol 2011;26:44-48.

28. Galan MV, Kishan AA, Silverman AL. Oral broccoli sprouts for the treatment of Helicobacter pylori infection: a preliminary report. Dig Dis Sci 2004;49:1088-1090.

29. Sato K, Kawakami N, Ohtsu T, et al. Broccoli consumption and chronic atrophic gastritis among Japanese males: an epidemiological investigation. Acta Med Okayama 2004;58:127-133.

30. Yang DH, Bom HS, Joo YE, Choi SK, Rew JS, Yoon CM. Gastric juice ammonia vs CLO test for diagnosis of Helicobacter pylori infection. Dig Dis Sci 1995;40:1083-1086.

31. Kearney DJ, Ritchie K, Peacock JS. Gastric-juice ammonia assay for diagnosis of Helicobacter pylori infection and the relationship of ammonia concentration to gastritis severity. Am J Gastroenterol 2000;95:3399-3403.

32. Mehta A, Singh S, Ganguly NK. Impairment of intestinal mucosal antioxidant defense system during Salmonella typhimurium infection. Dig Dis Sci 1998;43:646-651.

33. Jung HK, Lee KE, Chu SH, Yi SY. Reactive oxygen species activity, mucosal lipoperoxidation and glutathione in Helicobacter pyloriinfected gastric mucosa. J Gastroenterol Hepatol 2001;16:13361340. 
34. Shirin H, Pinto JT, Liu LU, Merzianu M, Sordillo EM, Moss SF. Helicobacter pylori decreases gastric mucosal glutathione. Cancer Lett 2001;164:127-133.
35. Matthews GM, Tivey D, Cummins AG, Butler RN. Cellular mucosal defense is attenuated with chronicity of Helicobacter pylori infection. Dig Dis Sci 2004;49:1109-1115. 